\title{
VC-sets and generic compact domination
}

\author{
Pierre Simon* \\ CNRS, Université Lyon 1
}

\begin{abstract}
Let $X$ be a closed subset of a locally compact second countable group $G$ whose family of translates has finite VC-dimension. We show that the topological border of $X$ has Haar measure 0. Under an extra technical hypothesis, this also holds if $X$ is constructible. We deduce from this generic compact domination for definably amenable NIP groups.
\end{abstract}

Let $G$ be a locally compact second countable group and fix a Haar measure $\mu$. Let $\mathcal{F} \subseteq \mathfrak{P}(G)$ be a family of subsets of $G$. We say that $\mathcal{F}$ has finite VC-dimension if for some $n$, for every subset $A \subset G$ of size $n$, we have $\mathcal{F} \cap$ $A \neq \mathfrak{P}(A)$, where $\mathcal{F} \cap A=\{S \cap A: S \in \mathcal{F}\}$. This notion has been well studied (in fact for families of subsets of arbitrary measure spaces). A number of combinatorial and measure theoretic properties are known: most notably families of finite VC-dimension have $\epsilon$-approximations (Fact 1.1). See [Mat02, Chapter 10] for more information. In the first section of this paper, we assume that the family $\mathcal{F}$ is stable under translation and our aim is to show, under further technical conditions, that the sets in $\mathcal{F}$ cannot be too fractal-like. For this, we might as well assume that $\mathcal{F}$ is exactly the family of translates of some set $X \subseteq G$. Our main theorem is the following.

Theorem 0.1. Let $X \subseteq G$ and assume that the family of left-translates of $X$ has finite VC-dimension. Assume furthermore that either $X$ is closed or that the following holds for both $Y=X$ and $Y=G \backslash X$ :

$Y$ is $F_{\sigma}$ and for all $x \in Y$ and neighborhood $U$ of $x, \mu(U \cap Y)>0$. $\underline{\text { Then }}$ the topological border $\partial X$ of $X$ has Haar measure 0.

\footnotetext{
*Partially supported by the European Research Council under the European Unions Seventh Framework Programme (FP7/2007-2013) / ERC Grant agreement no. 291111. Partially supported by ValCoMo (ANR-13-BS01-0006)
} 
This theorem has a precise motivation coming from model theory: In the second part of the paper, we use it to prove the generic compact domination conjecture for definably amenable NIP groups. To explain this, we now assume that the reader is familiar with model theory and in particular NIP theories.

Let $T$ be an NIP theory and $G$ a definable group in $T$. As usual, we denote by $G^{00}$ the smallest type-definable subgroup of $G$ of bounded index (which exists by NIP). We say that $G$ is definably amenable if it admits a $G$ invariant finitely additive probability measure on definable subsets of $G$. This notion was first studied in [HPP08, [HP11 and more systematically explored in [CS15]. The papers [HPP08] and [HP11] study in particular $f s g$ groups which are definably amenable groups which enjoy stronger stable-like properties. The typical example of an fsg group is a definably compact group in an o-minimal theory. Such a group has a unique invariant measure $\mu$. Let now $S \subset S_{G}(\mathcal{U})$ be the set of global generic types of $G$, equivalently (under the fsg assumption) the set of types $p \in S_{G}(\mathcal{U})$ which are $G^{00}$-invariant. Let $X$ be a definable subset of $G$ and let $h$ be the Haar measure on the compact group $G / G^{00}$. The generic compact domination conjecture from [HP11] says that for $h$-almost every coset $g G^{00}$ of $G^{00}$, the set $S \cap g G^{00}$ lies either entirely inside $X$ or entirely outside $X$.

A proof of this conjecture was announced in [HPS13], but there is a mistake there: the proof of Theorem 4.3 is flawed. We will give here the first (hopefully) correct proof of the conjecture. In fact, we prove a more general statement that holds for all definably amenable groups, making use of the work done in [CS15].

We also deduce as a corollary the following result: If $G$ is definably amenable NIP and $\mu$ is a global measure which is $G^{00}$-invariant and $G\left(M_{0}\right)$-invariant for a small model $M_{0}$, then $\mu$ is actually $G$-invariant.

Thanks to the referee for a number of helpful remarks.

\section{Preliminaries}

Let $G$ be a topological space. A constructible set $X \subseteq G$ is a finite boolean combination of closed sets. If $X \subseteq G$, we let $\partial X$ denote the border of $X$ : $\partial X=\bar{X} \backslash \stackrel{\circ}{X}=\bar{X} \cap \overline{X^{c}}$. We also let $X^{e x t}$ denote the exterior of $X$, that is the interior of $X^{c}$.

We now recall some facts about VC-dimension without proofs. A good reference on the subject is [Mat02, Chapter 10]. 
Let $X$ be any set and $\mathcal{F} \subseteq \mathfrak{P}(X)$ a family of subsets of $X$. For $X_{0} \subseteq X$, we write $\mathcal{F} \cap X_{0}=\left\{S \cap X_{0}: S \in \mathcal{F}\right\}$ and we say that $X_{0}$ is shattered by $\mathcal{F}$ if $\mathcal{F} \cap X_{0}=\mathfrak{P}\left(X_{0}\right)$.

We say that the family $\mathcal{F}$ has VC-dimension $n$ if it shatters some set of size $n$, but no set of size $n+1$. We say that it has infinite VC-dimension if it does not have finite VC-dimension, that is if for every $n$, some subset $X_{n} \subseteq X$ of size $n$ is shattered by $\mathcal{F}$. The exact value of the VC-dimension will not be important to us here. What is important is the dichotomy between finite and infinite VC-dimension.

One can also define the dual VC-dimension of $\mathcal{F}$ as the largest integer $n$ (or $\infty$ if there is none) such that there are $X_{1}, \ldots, X_{n} \in \mathcal{F}$ for which all the $2^{n}$ cells in the Venn diagram they generate are non-empty. An easy but important observation is that $\mathcal{F}$ has finite VC-dimension if and only if it has finite dual VC-dimension (though the two may not coincide). We will use this freely in this text.

The only theorem we will need about families of finite VC-dimension is the following fundamental fact (first proved in [VC71]). In the statement, and later in the text, $\operatorname{Av}\left(x_{1}, \ldots, x_{n} ; S\right)$ stands for $\frac{1}{n}\left|\left\{i \leq n: x_{i} \in S\right\}\right|$.

Fact 1.1 ( $\epsilon$-approximations). For any $k>0$ and $\varepsilon>0$ there is $N \in \mathbb{N}$ satisfying the following.

If $(X, \mu)$ is a probability space, and $\mathcal{F}$ a family of subsets of $X$ of $V C$ dimension $\leq k$ such that:

1. every set in $\mathcal{F}$ is measurable;

2. for each $n$, the function $f_{n}: X^{n} \rightarrow[0,1]$ given by

$$
\left(x_{1}, \ldots, x_{n}\right) \mapsto \sup _{S \in \mathcal{F}}\left|\operatorname{Av}\left(x_{1}, \ldots, x_{n} ; S\right)-\mu(S)\right|
$$

is measurable;

3. for each $n$, the function $g_{n}: X^{2 n} \rightarrow[0,1]$

$$
\left(x_{1}, \ldots, x_{n}, y_{1}, \ldots, y_{n}\right) \mapsto \sup _{S \in \mathcal{F}}\left|\operatorname{Av}\left(x_{1}, \ldots, x_{n} ; S\right)-\operatorname{Av}\left(y_{1}, \ldots, y_{n} ; S\right)\right|
$$

is measurable.

Then there are some $x_{1}, \ldots, x_{N} \in X$ (possibly with repetitions) such that for any $S \in \mathcal{F}$,

$$
\left|\operatorname{Av}\left(x_{1}, \ldots, x_{N} ; S\right)-\mu(S)\right|<\epsilon .
$$


We check now that the measurability conditions are satisfied when working with the family of translates of a single measurable set in a locally compact group.

Proposition 1.2. Let $G$ be a second countable locally compact group equipped with a Haar measure $\mu$. Let $U, X \subseteq G$ be Borel sets. Then the family $\mathcal{F}=$ $\{g X: g \in U\}$ of $U$-translates of $X$ satisfies the assumptions 1, 2, 3 in Fact 1.1 with respect to $(G, \mu)$.

Proof. Assumption 1 is clear since Borel sets are measurable.

Define $f_{n}$ and $g_{n}$ as in assumptions 2 and 3 and we need to show that $f_{n}$ and $g_{n}$ are measurable.

Since all the elements of $\mathcal{F}$ have the same measure $\mu(X)$, we have

$$
f_{n}\left(x_{1}, \ldots, x_{n}\right)=\sup _{g \in U}\left|\operatorname{Av}\left(x_{1}, \ldots, x_{n} ; g X\right)-\mu(X)\right| .
$$

Note that $\operatorname{Av}\left(x_{1}, \ldots, x_{n} ; g X\right)$ can take only finitely many values. It is then enough to show that for a fixed $I \subseteq n$, the set

$$
A_{I}=\left\{\left(x_{1}, \ldots, x_{n}\right) \in G^{n} \mid \text { for some } g \in U, x_{i} \in g X \Leftrightarrow i \in I\right\}
$$

is measurable. But we can write $A_{I}$ as the projection of $A_{I}^{\prime} \subseteq G^{n+1}$ where $A_{I}^{\prime}$ is the intersection of $\left\{\left(g, x_{1}, \ldots, x_{n}\right): g^{-1} x_{i} \in X, g \in U\right\}$ for $i \in I$ and $\left\{\left(g, x_{1}, \ldots, x_{n}\right): g^{-1} x_{i} \notin X, g \in U\right\}$ for $i \notin I$. As group multiplication is continuous and both $X$ and $U$ are Borel, those sets are Borel as well. Hence $A_{I}$ is analytic. Now $G$ is a Polish space ([Kec95, 9.A]) and analytic subsets of Polish spaces are universally measurable (that is measurable for any $\sigma$ finite Borel measure; see e.g. [Kec95, Theorem 29(7)]). In particular they are measurable with respect to the Haar measure $\mu$.

Finally, measurabilty of the function $g_{n}$ follows at once from measurability of the sets

$$
B_{I}=\left\{\left(x_{1}, \ldots, x_{n}, x_{n+1}, \ldots, x_{2 n}\right) \in G^{2 n} \mid \text { for some } g \in U, x_{i} \in g X \Leftrightarrow i \in I\right\},
$$

where now $I \subseteq 2 n$.

\section{VC-sets in locally compact groups}

In this section, we fix a second countable locally compact group $G$ and a leftinvariant Haar measure $\mu$ on it. 
For any integer $k$ and set $A$, we let ${ }^{k} A$ denote the set of functions from $k=\{0, \ldots, k-1\}$ to $A$.

Definition 2.1. A set $X \subseteq G$ is called a $V C$-set if the family $\{g X: g \in G\}$ of left translates of $X$ has finite VC-dimension.

Definition 2.2. A pair $\left(V_{0}, V_{1}\right)$ of disjoint open subsets of $G$ is called a tame pair if there is a maximal $n$ for which we can find $g_{0}, \ldots, g_{n-1}$ and $\left(x_{\eta}: \eta \in\right.$ $\left.{ }^{n}\{0,1\}\right)$ in $G$ such that for all $k<n$ and $\eta, g_{k} x_{\eta} \in V_{\eta(k)}$.

In particular, if $X$ is a VC-set, then $\left(\stackrel{\circ}{X}, X^{e x t}\right)$ is a tame pair (using VCduality). This terminology is inspired from similar notions arising in the study of tame dynamical systems (see e.g. Hua06]).

We recall the following well-known theorem of Steinhaus which holds in any locally compact group (see e.g. [Str72] for a short proof).

Fact 2.3 (Steinhaus). Let $X \subseteq G$ be a measurable set of positive Haar measure. Then the set $X X^{-1}=\left\{x y^{-1}: x, y \in X\right\}$ contains a neighborhood of the identity.

We now prove the main technical theorem of this paper.

Theorem 2.4. Let $\left(V_{0}, V_{1}\right)$ be a tame pair in $G$, then $\mu\left(\overline{V_{0}} \cap \overline{V_{1}}\right)=0$.

Proof. Set $\delta=\overline{V_{0}} \cap \overline{V_{1}}$ and assume that $\mu(\delta)>0$. Let $\Delta=\{x \in \delta: \mu(U \cap \delta)>0$ for all neighborhoods $U$ of $x$ \}.

Let $\left(O_{i}: i \in \mathbb{N}\right)$ be a countable base of open sets of $G$. For any $x \in \delta \backslash \Delta$, there is some $i(x) \in \mathbb{N}$ such that $x \in O_{i(x)}$ and $\mu\left(O_{i(x)} \cap \delta\right)=0$. Then $\delta \backslash \Delta=\bigcup_{x \in \delta \backslash \Delta} O_{i(x)} \cap \delta$ is a countable union of measure 0 sets and hence has measure 0. In particular $\mu(\Delta)>0$ and for $x \in \Delta$ and $U$ a neighborhood of $x$, $\mu(U \cap \Delta)>0$.

Fix some integer $n$ and we will construct inductively points $\left(g_{i}: i<n\right)$ in $G$ and $\left(x_{\eta}: \eta \in{ }^{k}\{0,1\}\right)$ in $\Delta, k \leq n$, such that

$$
\forall l \forall \eta \in{ }^{l}\{0,1\} \forall k<l \quad g_{k} x_{\eta} \in V_{\eta(k)} .
$$

This will contradict the fact that $\left(V_{0}, V_{1}\right)$ is tame.

Step 0: By Steinhaus' theorem, let $U$ be a neighborhood of 1 such that $U \subseteq$ $\overline{\Delta \Delta^{-1}}$. Let $x_{\langle 0\rangle} \in \Delta$ be any point. Take some $g_{0}^{0} \in U$ such that $g_{0}^{0} x_{\langle 0\rangle} \in V_{0}$. By definition of $U$, there is $x_{\langle 1\rangle} \in \Delta$ such that $g_{0}^{0} x_{\langle 1\rangle} \in \Delta$. Then there is some 
$g_{0}^{1} \in G$ such that $g_{0}^{1} g_{0}^{0} x_{\langle 1\rangle} \in V_{1}$ and $g_{0}^{1} g_{0}^{0} x_{\langle 0\rangle} \in V_{0}$ (because $V_{0}$ is open and $\left.g_{0}^{0} x_{\langle 1\rangle} \in \overline{V_{1}}\right)$. Set $g_{0}=g_{0}^{1} g_{0}^{0}$ and we finish step 0 .

Step $l$ : Assume that we have defined $\left(x_{\eta}: \eta \in{ }^{l}\{0,1\}\right)$ and $\left(g_{i}: i<l\right)$. As $\overline{V_{0}, V_{1}}$ are open, we can find open neighborhoods $W_{\eta}$ of $x_{\eta}$ such that for any $x^{\prime} \in W_{\eta}$ and $k<l$, we have $g_{k} x^{\prime} \in V_{\eta(k)}$. Set $X_{\eta}=W_{\eta} \cap \Delta$. By construction of $\Delta, X_{\eta}$ has positive measure. Apply Steinhaus' theorem to find some open neighborhood $U_{l}$ of 1 such that $U_{l} \subseteq X_{\eta} X_{\eta}^{-1}$ for each $\eta$.

Now enumerate ${ }^{l+1}\{0,1\}$ in an arbitrary order as $\left(\eta_{0}, \ldots, \eta_{2^{l+1}-1}\right)$. We define elements $\left(g_{l}^{i}: i<2^{l+1}\right)$ and $\left(x_{\eta_{i}}: i<2^{l+1}\right)$ inductively such that for all $j \leq i<2^{l+1}$ :

$\bullet_{0} g_{l}^{i} \cdots g_{l}^{0} \in U_{l}$

$\bullet_{1} g_{l}^{i} \cdots g_{l}^{0} x_{\eta_{j}} \in V_{\eta_{j}(l)}$;

$\bullet_{2}$ for $\lambda \in{ }^{l}\{0,1\}$ and $\alpha \in\{0,1\}, x_{\lambda^{\wedge} \alpha} \in X_{\lambda}$.

Once this is done, set $g_{l}=g_{l}^{2^{l+1}-1} \cdots g_{l}^{0}$ to finish step $l$ : condition $\bullet_{1}$ ensures that multiplication by $g_{l}$ sends the $x_{\eta}$ 's in the right $V_{i}$ and $\bullet_{2}$ ensures this for multiplication by all previous $g_{k}$ 's.

Assume that we have achieved this for all $j<i$. Set $\eta=\eta_{i}, \alpha=\eta(l)$ and $\lambda \in{ }^{l}\{0,1\}$ such that $\eta=\lambda^{\wedge} \alpha$. Let $x_{\eta} \in X_{\lambda}$ such that $g_{l}^{i-1} \cdots g_{l}^{0} x_{\eta} \in \Delta$ (possible by $\bullet_{0}$ and construction of $U_{l}$ ). Then find some $g_{l}^{i} \in G$ small enough such that:

$$
\begin{aligned}
& g_{l}^{i} \cdots g_{l}^{0} \in U_{l} ; \\
& g_{l}^{i} \cdots g_{l}^{0} x_{\eta_{j}} \in V_{\eta_{j}(l)} \text { for each } j<i \\
& g_{l}^{i} \cdots g_{l}^{0} x_{\eta} \in V_{\alpha} .
\end{aligned}
$$

This is possible as $U_{l}, V_{0}$ and $V_{1}$ are open and $g_{l}^{i-1} \cdots g_{l}^{0} x_{\eta} \in \overline{V_{\alpha}}$. This finishes the construction.

Lemma 2.5. Let $X \subseteq G$ be a VC-set which is $F_{\sigma}$. If $\mu(X)>0$, then $X$ has non-empty interior.

Proof. Fix some compact symmetric neighborhood $U$ of the identity. Take a compact set $B^{\prime}$ such that $\mu\left(X \cap B^{\prime}\right)=: \epsilon>0$. Let $B=U B^{\prime}$. Then $B$ is compact and hence has finite measure. Note that for each $g \in U$, we have $g X \cap B \supseteq g\left(X \cap B^{\prime}\right)$, hence $\mu(g X \cap B) \geq \mu\left(X \cap B^{\prime}\right)=\epsilon$.

Let $\mathcal{F}=\{g X \cap B: g \in U\}$ be the family of $U$-translates of $X$ intersected by $B$ so as to remain in a space of finite measure. It has finite VC-dimension as $X$ is a VC-set. Since all the elements of $\mathcal{F}$ have measure $>\epsilon$, by Fact 1.1 (and Proposition 1.2) applied to the Haar measure restricted to $B$, there is a finite set of points $\left\{x_{0}, \ldots, x_{n-1}\right\}$ meeting every translate $g X, g \in U$. Then for each 
$g \in U$, there is $k<n$ such that $g^{-1} x_{k} \in X$. In other words, $U \subseteq \bigcup_{i<n} X \cdot x_{i}^{-1}$. As each of the sets $X \cdot x_{i}^{-1}$ is $F_{\sigma}$, by the Baire property one of them must have non-empty interior and then so does $X$.

Theorem 2.6. Let $F \subseteq G$ be a closed $V C$-set. Then $\mu(\partial F)=0$.

Proof. Assume for a contradiction that $\mu(\partial F)>0$ and define $\Delta F=\{x \in \partial F$ : $\mu(U \cap \partial F)>0$ for all neighborhoods $U$ of $x\}$. Then as in the proof of Theorem 2.4. $\mu(\partial F \backslash \Delta F)=0$ and $\mu(\Delta F)>0$. Let $x \in \Delta F$ and let $U^{\prime}$ be a compact neighborhood of $x$. Then $r:=\mu\left(F \cap U^{\prime}\right) \geq \mu\left(\Delta F \cap U^{\prime}\right)>0$. Take also $W$ a symmetric compact neighborhood of 1 and let $U=W U^{\prime}$, so that for each $g \in W, \mu(g F \cap U) \geq r$. The family $\{g F \cap U: g \in W\}$ has finite VC-dimension and is included in the finite measure space $U$. We conclude as in Lemma 2.5. by the VC-theorem, there is a finite set $\left\{x_{0}, \ldots, x_{n-1}\right\} \subseteq U$ meeting every $g F$, $g \in W$. Then for some $i, F x_{i}^{-1} \cap W$ has non-empty interior. Hence $F \cap W U$ has non-empty interior. As we can take $U$ and $W$ arbitrarily small, $W U$ is an arbitrarily small neighborhood of $x$, and hence $x \in \overline{\stackrel{\circ}{F}}$.

The assumption on $F$ implies that $\left(\stackrel{\circ}{F}, F^{c}\right)$ is a tame pair. By the previous theorem, $\delta:=\overline{\bar{F}} \cap \overline{F^{c}}$ has measure 0. But we have shown that $\Delta F \subseteq \delta$, hence also $\Delta F$ has measure 0 .

In the statement above, one cannot replace closed by constructible (finite boolean combination of closed sets). Here is a counterexample in $(\mathbb{R},+)$ : take $K \subseteq \mathbb{R}$ a Cantor set of positive Lebesgue measure. Write $\mathbb{R} \backslash K$ as a countable union of disjoint intervals $\left(\left(a_{i}, b_{i}\right): i \in \mathbb{N}\right)$. For each $i \in \mathbb{N}$ consider an increasing sequence $\left(c_{k}^{i}: k \in \mathbb{Z}\right)$ in $\left(a_{i}, b_{i}\right)$ whose limits at $\pm \infty$ are respectively $a_{i}$ and $b_{i}$. Let $X$ be the union of all those sequences. Then $X$ is constructible, indeed locally closed, because it is discrete. Also $\partial X=\bar{X}=K \cup X$. In particular $\mu(\partial X)>0$. To make $X$ into a VC-set simply choose the points $c_{k}^{i}$ so that the map $X \times X \rightarrow \mathbb{R},(x, y) \mapsto y-x$ is injective. Then it is easy to see that the family of translates of $X$ cannot shatter a set of size 3 .

However, if $X$ is a finite boolean combination of closed VC-sets, then the theorem does hold for $X$, because the border of a boolean combination is included in the union of the borders of the sets in question.

We now state a second version of the theorem which applies in particular for constructible sets, under an extra assumption.

Theorem 2.7. Let $X$ be a VC-set and assume that the following condition holds both for $Y=X$ and $Y=X^{c}$ : 
$Y$ is $F_{\sigma}$ and for all $x \in Y$ and neighborhood $U$ of $x, \mu(U \cap Y)>0$. Then $\mu(\partial X)=0$.

Proof. As $X$ is a VC-set, the pair $\left(\stackrel{\circ}{X}, X^{e x t}\right)$ is tame. By Theorem 2.4, it is enough to show that $\partial X=\bar{X} \cap \overline{X^{e x t}}$. Let $x \in \partial X$. By symmetry of the roles of $X$ and $X^{c}$ it is enough to show that $x \in \overline{\dot{X}}$. Assume first that $x \in X$. Take $U$ a neighborhood of $x$ of finite measure. Then $X \cap U$ has positive measure by assumption. By the same reasoning as in Theorem 2.6, we conclude that $X \cap U$ has non-empty interior, which gives what we want. Now assume that $x \in X^{c}$. Let $U$ be a neighborhood of $x$. As $x \in \partial X$, there is some $x^{\prime} \in U \cap X$. Then by the argument in the first case, $x^{\prime}$ is in $\bar{X}$. As $U$ was arbitrary, also $x$ is in $\overline{\dot{\circ}}$.

\section{Generic compact domination}

In this section, we apply the previous results to prove a conjecture in model theory concerning definably amenable NIP groups. We assume familiarity with model theory and in particular NIP theories.

Let $T$ be an NIP theory and $M_{0} \models T$ a small model. Let also $G$ be an $M_{0}$-definable group. We assume that $G$ is definably amenable, which means that there exists a $G$-invariant Keisler measure on $G$. See [Sim15, Chapter 8], CS15] for background on this notion.

Recall that $G$ admits a smallest type-definable group of bounded index $G^{00}$ and that the quotient $K=G / G^{00}$ equipped with the logic topology is a compact Hausdorff group. We let $h$ denote the normalized Haar measure on it.

Recall the notion of f-generic type (in the sense of [CS15]): a global type $p$ concentrating on $G$ is f-generic if for any $\phi(x) \in p$, there is a small model $M$ such that no (left-)translate of $\phi(x)$ forks over $M$. Equivalently, $p$ is $G^{00}$ invariant (by left translations). Such a type gives rise to an invariant measure $\mu_{p}$ on $G$ defined by $\mu_{p}(\phi)=h\left(\left\{\bar{g} \in G / G^{00}: \bar{g} \cdot p \vdash \phi(x)\right\}\right)$ for any definable subset $\phi(x)$ of $G$. If $p$ is weakly-random for $\mu_{p}$-which means that $\mu_{p}(X)>0$ for any $X \in p$-then we call $p$ an almost periodic type. This is equivalent to asking that $\overline{G \cdot p}$, the closure of the orbit of $p$ in $S(\mathcal{U})$, is a minimal $G$-invariant closed subset of $S(\mathcal{U})$. All this is explained in CS15.

Let $p$ be an almost periodic type concentrating on $G^{00}$, and let $\pi: \overline{G \cdot p} \rightarrow$ $G / G^{00}$ be the canonical projection. For $\phi(x)$ a definable set of $G$-which we 
identify with the corresponding clopen subset of $S(\mathcal{U})$ - let $X=d_{p}(\phi) \subseteq G / G^{00}$ be the set of $\bar{g} \in G / G^{00}$ such that $\bar{g} \cdot p \vdash \phi(x)$. Finally, define

$$
E_{\phi}=\left\{\bar{g} \in G / G^{00}: \pi^{-1}(\bar{g}) \cap \phi \neq \emptyset \text { and } \pi^{-1}(\bar{g}) \cap \neg \phi \neq \emptyset\right\} .
$$

The following is shown in CS15.

Fact 3.1. We have the inclusion $E_{\phi} \subseteq \partial X$ and the set $E_{\phi}$ is a closed meager set.

We can now state the compact domination theorem.

Theorem 3.2. The set $E_{\phi}$ has Haar measure 0 .

Proof. First we show that we may restrict to the case where $L$ is countable. Let $L_{0}$ be a countable sublanguage containing $\phi$ and the formulas defining $G$ and its group structure. Let $G_{L_{0}}^{00}$ be $G^{00}$ in the sense of $L_{0}$. We have a canonical surjection $\pi_{0}: G / G^{00} \rightarrow G / G_{L_{0}}^{00}$. Let $E_{\phi}^{0}$ be the set $E_{\phi}$ in the sense of $L_{0}$. Then $E_{\phi} \subseteq \pi_{0}^{-1}\left(E_{\phi}^{0}\right)$. Also the Haar measure on $G / G_{L_{0}}^{00}$ is the pushforward of the Haar measure on $G / G^{00}$ (by uniqueness). It is now enough to show that $E_{\phi}^{0}$ has Haar measure 0 . Therefore we may replace $L$ by $L_{0}$ and assume that $L$ is countable. Then $G / G^{00}$ is a second countable compact group.

Claim 1: $X=d_{p}(\phi)$ is a constructible VC-set in $G / G^{00}$.

Proof: The fact that $d_{p}(\phi)$ is constructible was shown in CS15, it easily follows from Borel-definability of invariant types in NIP theories (Proposition 2.6 in [HP11]). To see that $d_{p}(\phi)$ is a VC-set, let $\left\{x_{0}, \ldots, x_{n-1}\right\} \subseteq K$ be a finite set shattered by the family of translates of $d_{p}(\phi)$. For $i \subseteq n$, let $y_{i}$ be such that $x_{k} \in y_{i} \cdot d_{p}(\phi) \Longleftrightarrow k \in i$.

For each $k<n, i \subseteq n$, pick representatives $g_{k}$ of $x_{k}$ and $h_{i}$ of $y_{i}$ in $G(\mathcal{U})$. Finally, let $a$ realize $p$ over all those points. We see that

$$
\models \phi\left(h_{i}^{-1} g_{k} a\right) \Longleftrightarrow k \in i .
$$

This shows that $n$ is at most the VC-dimension of the formula $\psi(x y ; z)=$ $\phi\left(z^{-1} \cdot x \cdot y\right)$ and concludes the proof of the claim.

Claim 2: For $x_{*} \in X$ and $U \subseteq G / G^{00}$ an open neighborhood of $x_{*}, h(U \cap$ $X)>0$.

Proof: By construction of the topology on $G / G^{00}$, there is a definable set $\psi(x) \in L\left(M_{0}\right)$ such that $\pi^{-1}\left(\left\{x_{*}\right\}\right) \subseteq \psi(x) \subseteq \pi^{-1}(U)$. Take $g \in G(\mathcal{U})$ projecting to $x_{*}$. As $p$ concentrates on $G^{00}$, we have $\pi(g \cdot p)=x_{*}$ and $g \cdot p \vdash \psi(x)$. 
Therefore $g \cdot p \vdash \psi(x) \wedge \phi(x)$ and hence $\mu_{p}(\psi(x) \wedge \phi(x))>0$. This exactly means that $h\left(d_{p}(\psi) \cap d_{p}(\phi)\right)>0$. As $p$ concentrates on $G^{00}$, we have $d_{p}(\psi) \subseteq U$ and thus $h(U \cap X)>0$.

Replacing $\phi(x)$ by $\neg \phi(x)$ we obtain the same result for $X^{c}$. Now we can apply Theorem 2.7 to obtain $\mu(\partial X)=0$. Then also $\mu\left(E_{\phi}\right)=0$ by Fact 3.1 .

\section{1 $G\left(M_{0}\right)$-invariant measures}

We keep notations as above: $G$ is a definably amenable NIP group defined over some model $M_{0}$. If $\mu(x)$ is a measure over $M$, then the support of $\mu$ is the (closed) set of types $p \in S_{x}(M)$ such that $p \vdash \phi(x) \Longrightarrow \mu(\phi(x))>0$.

We will need the following facts. First an easy property of measures.

Fact 3.3 (CS15], Proposition 3.15). A global measure $\mu$ on $G$ is $G^{00}$-invariant if and only if all types in its support are f-generic.

Next a model-theoretic adaptation of Fact 1.1; here $\operatorname{Av}\left(p_{1}, \ldots, p_{n} ; \phi(x)\right)$ means $\frac{1}{n}\left|\left\{i: p_{i} \vdash \phi(x)\right\}\right|$.

Fact 3.4 (Sim15, Proposition 7.11). Let $\mu(x)$ be any Keisler measure over a model $M$. Let $\phi(x ; y) \in L$ and $f i x \epsilon>0$. Then there are types $p_{1}, \ldots, p_{n}$ in the support of $\mu$ such that for any $b \in M$,

$$
\left|\operatorname{Av}\left(p_{1}, \ldots, p_{n} ; \phi(x ; b)\right)-\mu(\phi(x ; b))\right|<\epsilon .
$$

Proposition 3.5. Let $p \in S_{G}(\mathcal{U})$ be a global f-generic type. Fix a formula $\phi(x) \in L(\mathcal{U})$ and $\epsilon>0$. Then there are $g_{1}, \ldots, g_{n} \in G\left(M_{0}\right)$ such that for any $g \in G(\mathcal{U})$

$$
\left|\mu_{p}(\phi(x))-\operatorname{Av}\left(g_{1} \cdot p, \ldots, g_{n} \cdot p ; \phi\left(g^{-1} x\right)\right)\right|<\epsilon .
$$

Proof. First we show that we may assume that $L$ is countable. Let $L_{0}$ be a countable sublanguage sufficient to define $G$ and $\phi(x)$. Then the reduct of $p$ to $L_{0}$ is also f-generic. Letting $G_{L_{0}}^{00}$ be $G^{00}$ in the sense of $L_{0}$, we have a canonical $\operatorname{map} \pi_{0}: G / G^{00} \rightarrow G / G_{L_{0}}^{00}$ and the Haar measure on $G / G_{L_{0}}^{00}$ is the pushforward of the Haar measure on $G / G^{00}$. One sees then that the measure $\mu_{p}$ in the sense of $L_{0}$ agrees with $\mu_{p}$ in the sense of $L$ for $L_{0}$-formulas. Hence we may take $L$ to be countable and then $G / G^{00}$ is a compact second countable group.

Define $X=d_{p}(\phi(x))$ as above, namely $X=\left\{\bar{g} \in G / G^{00}: \bar{g} \cdot p \vdash \phi(x)\right\}$. So we have $\mu_{p}(\phi(x))=h(X)$. Fix a left-invariant metric $d$ on $G / G^{00}$. For $r>0$, 
let $V_{0, r} \subseteq \AA^{\circ}$ be the set of points at distance $>r$ from $\left(\stackrel{\circ}{X}^{c}\right.$ and $V_{1, r} \subseteq X^{e x t}$ be the set of points at distance $>r$ from $\bar{X}$. Then $\stackrel{\circ}{X}=\bigcup_{r>0} V_{0, r}$ and hence there is $r>0$ such that $\mu\left(V_{0, r}\right)>\mu\left(X^{\circ}\right)-\epsilon$. Similarly, we can take $r$ so that $\mu\left(V_{1, r}\right)>\mu\left(X^{e x t}\right)-\epsilon$. Fix such an $r$ and for $i=0,1$, let $V_{i}=V_{i, r}$.

By regularity of the Haar measure, there are closed sets $C_{0} \subseteq V_{0}$ and $C_{1} \subseteq V_{1}$ such that $h\left(V_{i} \backslash C_{i}\right)<\epsilon$ for $i=0,1$. Let $\pi: G \rightarrow G / G^{00}$ be the canonical projection. One can find definable sets $\theta_{0}$ and $\theta_{1}$ such that $\pi^{-1}\left(C_{i}\right) \subseteq \theta_{i} \subseteq \pi^{-1}\left(V_{i}\right), i=0,1$. Let $q$ be any f-generic type concentrating on $G^{00}$ and define $X_{i}=d_{q}\left(\theta_{i}\right)$. Then we have $C_{i} \subseteq X_{i} \subseteq V_{i}$ and $X_{i}$ is a Borel VCset as in the proof of Theorem 3.2. By the VC-theorem 1.1 (and Proposition 1.2), we can find points $\bar{h}_{1}, \ldots, \bar{h}_{n} \in G / G^{00}$ such that

$$
\left|h\left(\bar{g} X_{i}\right)-\operatorname{Av}\left(\bar{h}_{1}, \ldots, \bar{h}_{n} ; \bar{g} X_{i}\right)\right| \leq \epsilon,
$$

for all $\bar{g} \in G / G^{00}$ and $i=0,1$.

Now $\pi\left(G\left(M_{0}\right)\right)$ is dense in $G / G^{00}$, hence we can find points $g_{k} \in G\left(M_{0}\right)$, $k \leq n$, such that $d\left(\bar{g}_{k}, \bar{h}_{k}\right)<r$, where $\bar{g}_{k}=\pi\left(g_{k}\right)$. Fix any $\bar{g} \in G / G^{00}$. Then if $\bar{h}_{k} \in \bar{g} X_{0}$, we have $\bar{g}_{k} \in \bar{g} X$ since $\bar{g} X_{0} \subseteq \bar{g} V_{0, r}$. Similarly, if $\bar{h}_{k} \in \bar{g} X_{1}$, then $\bar{g}_{k} \in \bar{g} X^{e x t}$.

We then have:

$$
\operatorname{Av}\left(\bar{h}_{1}, \ldots, \bar{h}_{n} ; \bar{g} X_{0}\right) \leq \operatorname{Av}\left(\bar{g}_{1}, \ldots, \bar{g}_{n} ; \bar{g} X\right) \leq 1-\operatorname{Av}\left(\bar{h}_{1}, \ldots, \bar{h}_{n} ; \bar{g} X_{1}\right) .
$$

By construction of the $\bar{h}_{k}$ 's, $\operatorname{Av}\left(\bar{h}_{1}, \ldots, \bar{h}_{n} ; \bar{g} X_{i}\right) \geq h\left(\bar{g} X_{i}\right)-\epsilon \geq h\left(\bar{g} V_{i}\right)-2 \epsilon$. By Theorem 3.2, $h(\stackrel{X}{X})=h(X)$, therefore $h\left(\bar{g} V_{0}\right) \geq h(\bar{g} X)-\epsilon$. Also $h\left(X^{\text {ext }}\right)=$ $1-h(X)$, hence $1-h\left(\bar{g} V_{1}\right) \leq h(\bar{g} X)+\epsilon$. Putting it all together, we see that

$$
h(\bar{g} X)-3 \epsilon \leq \operatorname{Av}\left(\bar{g}_{1}, \ldots, \bar{g}_{n} ; \bar{g} X\right) \leq h(\bar{g} X)+3 \epsilon,
$$

from which the result follows.

Corollary 3.6. Let $p \in S_{G}(\mathcal{U})$ be almost periodic (equivalently, $p$ is f-generic and weakly random for $\mu_{p}$ ), then $\overline{G\left(M_{0}\right) \cdot p}=\overline{G(\mathcal{U}) \cdot p}$.

Proof. Assume that $\overline{G\left(M_{0}\right) \cdot p} \subsetneq \overline{G(\mathcal{U}) \cdot p}$, then there is a formula $\phi(x)$ which is disjoint from the first set, but not from the second. But then $\mu_{p}(\phi(x))>0$ by almost periodicity which contradicts Proposition 3.5.

Corollary 3.7. The minimal flows in the dynamical system $\left(G\left(M_{0}\right), S_{G}\left(M_{0}\right)\right)$ are precisely the projections of the minimal flows in $\left(G(\mathcal{U}), S_{G}(\mathcal{U})\right)$. 
Proof. Let $\pi: S_{G}(\mathcal{U}) \rightarrow S_{G}\left(M_{0}\right)$ be the natural projection. Let first $Y \subseteq$ $S_{G}(\mathcal{U})$ be a minimal flow. So $Y$ has the form $\overline{G \cdot p}$ for some almost periodic type $p$. By the previous corollary, $G\left(M_{0}\right) \cdot p$ is dense in $Y$, hence also $G\left(M_{0}\right) \cdot \pi(p)$ is dense in $\pi(Y)$. As this is true for all $p \in Y, \pi(Y)$ is a minimal flow.

The converse is true without any assumptions on $T$ or $G$ : let $X \subset S_{G}\left(M_{0}\right)$ be a minimal flow under the action of $G\left(M_{0}\right)$. Let $p \in X$ be a type and let $\tilde{p}$ be an heir of $p$ over $\mathcal{U}$. Given $\phi(x) \in L\left(M_{0}\right)$ disjoint from $X$, we have that $p \vdash \neg \phi(g \cdot x)$ for any $g \in G\left(M_{0}\right)$ as $X$ is a flow. By the heir property, also $\tilde{p} \vdash \neg \phi(g \cdot x)$ for any $g \in G(\mathcal{U})$. This means that the orbit $G(\mathcal{U}) \cdot p$ lies entirely inside $\pi^{-1}(X)$, hence so does its closure $\overline{G(\mathcal{U}) \cdot p}$, which is a subflow. Let $Y$ be a minimal $G(\mathcal{U})$-subflow inside that closure. Then $\pi(Y) \subseteq X$ is a $G\left(M_{0}\right)$-subflow, but then $\pi(Y)=X$ by minimality of $X$.

The point of the following theorem is that - at least when $L$ is countablethe study of invariant measures on $G$, can be reduced to the study of invariant measures for the action of a countable group on a compact space (which is the situation for many theorems in topological dynamics). Here, the compact space would be the space of $G^{00}(\mathcal{U})$-invariant types and the countable group would be $G\left(M_{0}\right)$, for $M_{0}$ a countable model.

Theorem 3.8. Let $G$ be a definably amenable NIP group and let $\mu$ be a global $G^{00}(\mathcal{U})$-invariant and $G\left(M_{0}\right)$-invariant measure. Then $\mu$ is $G(\mathcal{U})$-invariant.

Proof. By Fact 3.3, the support of $\mu$ consists only of f-generic types. Fix a formula $\phi(x) \in L(\mathcal{U})$ and $\epsilon>0$. By Fact [3.4, there are f-generic types $p_{1}, \ldots, p_{n}$ such that for any $g \in G(\mathcal{U})$, we have

$$
\left|\mu(\phi(g x))-\operatorname{Av}\left(p_{1}, \ldots, p_{n} ; \phi(g x)\right)\right| \leq \epsilon
$$

Proposition 3.5 is stated for one f-generic type, but works just as well for finitely many types: instead of doing the proof for just one $X=d_{p}(\phi)$, we have finitely many $X^{i}=d_{p_{i}}(\phi)$; we do the same analysis for each of them separately and apply the $\mathrm{VC}$-theorem for all of them at once. This gives us points $g_{1}, \ldots, g_{m} \in G\left(M_{0}\right)$ such that for each $i \leq n$ and for each $g \in G(\mathcal{U})$,

$$
\left|\mu_{p_{i}}(\phi(g x))-\operatorname{Av}_{j}\left(g_{j} \cdot p_{i} ; \phi(g x)\right)\right| \leq \epsilon .
$$

By $G\left(M_{0}\right)$-invariance of $\mu$, we also have that for any $g$ and $j \leq m$,

$$
\left|\mu(\phi(g x))-\operatorname{Av}\left(g_{j} \cdot p_{1}, \ldots, g_{j} \cdot p_{n} ; \phi(g x)\right)\right| \leq \epsilon
$$


Hence averaging over all $p_{i}$ 's and $g_{j}$ 's we obtain

$$
\left|\frac{1}{n} \sum_{i \leq n} \mu_{p_{i}}(\phi(g x))-\mu(\phi(g x))\right| \leq 2 \epsilon .
$$

and in particular

$$
\left|\frac{1}{n} \sum_{i \leq n} \mu_{p_{i}}(\phi(x))-\mu(\phi(x))\right| \leq 2 \epsilon .
$$

As the measure on the left-hand side is $G$-invariant, we deduce $\mid \mu(\phi(g x))-$ $\mu(\phi(x)) \mid \leq 4 \epsilon$, hence $\mu$ is $G$-invariant.

\section{2 fsg groups}

An NIP group $G$ is $f s g$ if there is some type $p \in S_{G}(\mathcal{U})$ and a small model $M_{0}$ such that all translates of $p$ are finitely satisfiable in $M_{0}$. It is shown in HP11 that such a group admits a unique invariant measure $\mu$, in particular it is definably amenable. Also, any set of positive measure is generic (finitely many translates cover the group). Let $p$ be a generic type, equivalently a type weakly-random for $\mu$, then $\overline{G \cdot p}$ is exactly the set of all generic types. Also, f-generic formulas and generic formulas coincide ([HPS12] or [CS15]). We let

$\pi: \overline{G \cdot p} \rightarrow G / G^{00}$ be the canonical projection. Theorem 3.2 gives us the following.

Corollary 3.9. Let $G$ be an $f s g$ group and let $S \subset S_{G}(\mathcal{U})$ be the set of generic types of $G$. Let $\phi$ be a definable subset of $G$ and define

$$
E_{\phi}=\left\{\bar{g} \in G / G^{00}: \pi^{-1}(\bar{g}) \cap \phi \neq \emptyset \text { and } \pi^{-1}(\bar{g}) \cap \phi^{c} \neq \emptyset\right\} .
$$

Then $E_{\phi}$ has Haar measure 0.

Corollary 3.10. Let $G$ be $f s g$, then there is a unique Keisler measure on $G$ which is $G^{00}$-invariant and lifts the Haar measure on $G / G^{00}$.

Proof. The fact that the previous corollary implies this one is already proved in [HPS13, Proposition 5.7], but we repeat the argument.

Let $\lambda$ be a global Keisler measure on $G$ which is $G^{00}(\mathcal{U})$-invariant and lifts the Haar measure on $G / G^{00}$. By Fact 3.3, the support of $\lambda$ is composed of f-generic - and thus generic - types, so $\lambda$ can be seen as a Borel measure on 
the set $S$ of global generic types. Compact domination then implies that it is entirely determined by its image $\pi_{*}(\lambda)$ on $G / G^{00}$ : Let $\phi(x)$ be a definable set. Then as $E_{\phi}$ has measure 0 , we must have $\lambda(\phi)=\pi_{*} \lambda(\pi(\phi))=h(\pi(\phi))$. This proves uniqueness of $\lambda$.

\section{References}

[CS15] Artem Chernikov and Pierre Simon. Definably amenable NIP groups. preprint, 2015.

[HP11] Ehud Hrushovski and Anand Pillay. On NIP and invariant measures. Journal of the European Mathematical Society, 13:1005-1061, 2011.

[HPP08] Ehud Hrushovski, Ya'acov Peterzil, and Anand Pillay. Groups, measures, and the NIP. J. Amer. Math. Soc., 21(2):563-596, 2008.

[HPS12] Ehud Hrushovski, Anand Pillay, and Pierre Simon. A note on generically stable measures and $f s g$ groups. Notre Dame Journal of Formal Logic, 53(4):599-605, 2012.

[HPS13] Ehud Hrushovski, Anand Pillay, and Pierre Simon. Generically stable and smooth measures in NIP theories. Trans. Amer. Math. Soc., 365:2341-2366, 2013.

[Hua06] Wen Huang. Tame systems and scrambled pairs under an abelian group action. Ergodic theory and dynamical systems, 26:1549-1567, 2006.

[Kec95] Alexander S Kechris. Classical descriptive set theory, volume 156. Springer-Verlag New York, 1995.

[Mat02] Jiri Matousek. Lectures on Discrete Geometry. Springer-Verlag New York, Inc., Secaucus, NJ, USA, 2002.

[Sim15] Pierre Simon. A Guide to NIP theories. Lecture Notes in Logic. Cambridge University Press, 2015.

[Str72] Karl Stromberg. An elementary proof of steinhaus's theorem. Proceedings of the American Mathematical Society, 36(1):308, 1972. 
[VC71] V. N. Vapnik and A. Ya. Chervonenkis. On the uniform convergence of relative frequencies of events to their probabilities. Theory Probab. Appl., 16(2):264-280, 1971. 